\title{
Sur les fonctions dérivées des fonctions mesurables.
}

\author{
Par \\ Stefan Banach (Léopol).
}

Le bat de cette Note est de démontrer que les fonctions dérivées de Dini d'une fonction $f(x)$ mesurable $(L)$ sont mesurables $(L)$. Notre démonstration permettra en même temps de prouver que les fonctions dérivées d'une fonction $f(x)$ bornée de classe a de Baire sont toujours de elasses $\leqslant a+21$ ).

Lemme. Prémisses: $f(x)$ est une fonction quelconque d'une variable réelle (mesurable ou non), $\delta$ - un nombre positif, $F(x)-$ la borne supérieure de tous les nombres

$$
f(x+h) \text { pour } 0 \leqslant h \leqslant \delta .
$$

Thèse: $F(x)$ est une fonction n'admettant que des discontinuités ordinaires (donc $F(x)$ est de classe $\leqslant 1$ de M. Baire).

Démonstration. Désignons par $B(\alpha, \beta)$ la borne supérieure des nombres $f(x)$ pour $\alpha \leqslant x \leqslant \beta$. Soit $x_{0}$ un nombre réel douné, $x$ - un nombre réel, tel que

$$
0<x-x_{0}<\delta \text {. }
$$

L'intervalle $(x, x+\delta)$ est donc une somme d'intervalles $\left(x, x_{0}+\delta\right)$ et $\left(x_{0}+\delta, x+\delta\right):$ par conséquent

$$
F(x)=B(x, x+\delta)=\operatorname{Max}\left[B\left(x, x_{0}+\delta\right), B\left(x_{0}+\delta, x+\delta\right)\right],
$$

Max $[a, b]$ désignant le plus grand de deux nombres $a, b$ (ou leur valeur commune).

1) Co théorème a été signalé (pour les fonctions $f(x)$ bornées ou non, do classe a) par M, Sierpitaski: v. ce volume, p. 137. 
Or, quand $x$ décroit, $B\left(x, x_{0}+\delta\right)$ ne peut éridemment diminuer et $B\left(x_{0}+\delta, x+\delta\right)$ ne peut augmenter: donc $B\left(x, x_{0}+\delta\right)$ et $B\left(x_{0}+\delta, x+\delta\right)$ ont des limites quand $x$ tend en décroissant vers $x$, donc, d'après (1), aussi $F(x)$. Donc la limite $F\left(x_{0}+0\right)$ est déterminée. De même on démontre que la limite $F\left(x_{0}-0\right)$ est déterminée. Notre lemme est ainsi démontré.

Corollaire I. Soit $F(x, a, b)(b>a>0)$ la borne supérieure de tous les nombres

$$
f(x+h) \text { pour } a \leqslant h \leqslant b .
$$

Thèse: $F(x, a, b)$ est une fonction de $x$ de classe $\leqslant 1$.

$\mathrm{D}$ èm. Nous a vons évidemment $F(x, a, b)=B(x+a . x+b)$. Or nous avons démontré (lemme I) que la fonction $\varphi(x)=B(x, x+b-a)$ est de classe $\leqslant 1$ : done aussi la fonction $F(x, a, b)=\varphi(x+a)$ est de classe $\leqslant 1$, c. q. f. $d$.

Corollarre II. Soit $f(x)$ une fonction de classe $a>0$ de M. Baire, $b>a>0, \varphi(x, a, b)$ la borne superieure des nombres

$$
f(x+h)-f(x) \text { pour } a \leqslant h \leqslant b .
$$

Thèse: $\varphi(x, a, b)$ est une fouction de classe $\leqslant \alpha$.

Pour démontrer notre corollaire il suffit de remarquer que $\varphi(x, a, b)=F(x . a, b)-f(x)$ et de faire appel au corollaire I et à la proposition qu'une différence de deux fonctions de classes $\leqslant \alpha$ est une fonction de classe $\leqslant \alpha$.

Pareillement on établit le.

Corollaire III. Soit $f(x)$ une fonction mesurable $(L)$ : $\varphi(x, a, b)$ ayant méme signification qu'au corollaire II, $\varphi(x, a, b)$ est une fonction mesurable $(L)$.

Soit maintenant $f(x)$ une fonction bornée, $a$ et $b>a$ deux nombres positifs. Désignons par $\Phi(x, a, b)$ la borne supérieure des nombres

$$
f(x+h)-f(x) \text { pour } a \leqslant h \leqslant b
$$

et par $\varphi(x, a, b)-$ la borne supérieure des nombres

$$
f(x+h)-f(x) \text { pour } a \leqslant h \leqslant b
$$

Soit $n$ un nombre naturel donné et posons

$$
a_{k}=a+\frac{k}{n}(b-a) \text { pour } k=0,1,2, \ldots
$$


$\Phi(x, a, b)$ sera évidemment le plus grand des' nombres

$$
\Phi\left(x, a_{k-1}, a_{k}\right) \quad(k=1,2, \ldots, n) .
$$

Nous avons, pour $\beta>\alpha>0$ :

$$
\begin{gathered}
f(x+h)-f(x) \leqslant \varphi(x, \alpha, \beta), \\
\frac{1}{\beta} \leqslant \frac{1}{h} \leqslant \frac{1}{\alpha},
\end{gathered} \mid \text { pour } \alpha \leqslant h \leqslant \beta
$$

done, pour $\varphi(x, \alpha, \beta) \geqslant 0$ :

$$
\frac{f(x+h)-f(x)}{h} \leqslant \frac{\varphi(x, \alpha, \beta)}{\alpha} \text { pour } \alpha \leqslant h \leqslant \beta
$$

et, pour $\varphi(x, \alpha, \beta) \leqslant 0$ :

$$
\frac{f(x+h)-f(x)}{h} \leqslant \frac{\varphi(x, \alpha, \beta)}{\beta} \text { pour } a \leqslant h \leqslant \beta .
$$

Par conséquent:

$$
\begin{cases} & \Phi(x, \alpha, \beta) \leqslant \frac{\varphi(x, \alpha, \beta)}{\alpha} \text { pour } \varphi(x, \alpha, \beta) \geqslant 0 \\ \text { et } \quad & \Phi(x, \alpha, \beta) \leqslant \frac{\varphi(x, \alpha, \beta)}{\beta} \text { pour } \varphi(x, \alpha, \beta) \leqslant 0 .\end{cases}
$$

Or

$$
f(x+h)-f(x) \leqslant h \Phi(x, \alpha, \beta) \text { pour } \quad \alpha \leqslant h \leqslant \beta,
$$

parsuite

(5) $\left\{\begin{array}{llll}\text { et } \quad & \varphi(x, \alpha, \beta) \leqslant \beta \Phi(x, \alpha, \beta) & \text { pour } & \Phi(x, \alpha, \beta) \geqslant 0 \\ & \varphi(x, \alpha, \beta) \leqslant \alpha \Phi(x, \alpha, \beta) & \text { pour } & \Phi(x, \alpha, \beta) \leqslant 0 .\end{array}\right.$

Les formules (4) et (5) démontrent que $\Phi(x, \alpha, \beta)$ et $\varphi(x, \alpha, \beta)$ ont toujours le même signe et que

et

$$
\frac{\varphi(x, \alpha, \beta)}{\beta} \leqslant \Phi(x, \alpha, \beta) \leqslant \frac{\varphi(x, \alpha, \beta)}{\alpha} \text { pour } \varphi(x, \alpha, \beta) \geqslant 0
$$

$$
\frac{\varphi(x, \alpha, \beta)}{\alpha} \leqslant \Phi(x . \alpha, \beta) \leqslant \frac{\varphi(x, \alpha, \beta)}{\beta} \text { pour } \varphi(x, \alpha, \beta) \leqslant 0
$$

La fonction $f(x)$ étant bornée et $M$ désignant un nombre tel que $|f(\xi)| \leqslant M$ (pour tout $\xi$ réel), nous avons $|f(x+h)-f(x)| \leqslant 2 M$, done

$$
\left|\Phi(c, \alpha, \beta) \frac{\varphi(x, \alpha, \beta)}{\alpha}\right| \leqslant|\varphi(x, \alpha, \beta)| \frac{\beta-\alpha}{\alpha \beta} \leqslant 2 M \frac{\beta-\alpha}{\alpha \beta},
$$


par conséquent, d'après (2):

$$
\left|\Phi\left(x, a_{k-1}, a_{k}\right)-\frac{\varphi\left(x . a_{k-1}, a_{k}\right)}{a_{k-1}}\right| \leqslant 2 M \frac{b-a}{n a^{2}}
$$

Soit $G(x, a, b, n)$ le plus grand des nombres

$$
\frac{\varphi\left(x, a_{k 1}, a_{k}\right)}{a_{k-1}} \quad(k=1,2, \ldots, n) .
$$

D'après (6) $(\Phi(x, a, b)$ étant le plus grand des nombres (3)) nous aurons évidemment

Par conséquent

$$
\Phi(x, a, b)-G(x, a, b, n) \mid \leqslant \frac{2 M(b-a)}{n a^{2}} .
$$

$$
\Phi(x, a, b)=\lim _{n=\infty} G(x, a, b, n),
$$

la suite à droite étant de plus convergente uniformément. Or nous avons évidemment

$$
\overline{f_{+}^{\prime}}(x)=\lim _{l=\infty} \lim _{k=\infty} \Phi\left(x, \frac{1}{k}, \frac{1}{l}\right) .
$$

Soit $f(x)$ une fonction bornée de classe $\alpha>0$ : d'après le corollaire II les fonctions (7) seront de classes $\leqslant \alpha$, done aussi $G(x, a, b, n)$. La suite (8) étant uniformément convergente, nous en concluons que $\Phi(x, a, b)$ est une fonction de classe $\leqslant \alpha$, donc, d'après $(9), \overline{f_{+}^{\prime}}(x)$ est de classe $\leqslant a+2$.

Si $f(x)$ (supposée bornée) était mesurable $(L)$, il en serait de même, d'après le corollaire III, avec les fonctions (7), et les formules (8) et (9) prouveraient que $\overline{f_{+}^{\prime}}(x)$ est mesurable $(L)$.

Soit maintenant $f(x)$ une fonction non bornée mesurable $(L)$, $f_{p}(x)$ - la fonction égale à $f(x)$ si $-p \leqslant f(x) \leqslant p$, à $p$ si $f(x)>p$ et à $-p$ si $f(x)<-p ; f_{p}(x)$ sera évidemment bornée et mesurable $(L)$ et il est aisé de voir que

$$
\overline{f_{+}^{\prime}}(x)=\lim _{p=\infty} \overline{f_{p+}^{\prime}}(x)
$$

d'où il résulte que $\overline{f_{+}^{\prime}}(x)$ est mesurable $(L)$, c. q. f. d.

Pour les fonctions non mesurables $(L)$ les dérivées de Dini peuvent être aussi bien mesurables $(L$, même $B$ ) que non mesurables, comme on le voit sans difficulté. 
Nous terminerons par deux théorèmes suivants:

Théorème I: Sila dérivé e $f_{+}^{\prime}(x)$ d'une fonction $f(x)$ est finie sauf dans un ensemble au plus dénombrable de points, $f(x)$ est une fonction mesurable $(B)$ (de classe $\leqslant 2$ ).

Dém. Soit $D$ l'ensemble (au plus dénombrable) où la derivée $+(x)$ est infinie. Considérons l'ensemble $E=\mathrm{E}(f(x) \geqslant A), A$ étant un nombre réel donné quelconque. Soit $x$ une limite d'une suite décroissante $x_{n}$ de nombres de $E$ et supposons que $x$ n'appartient pas à $E$. Nous aurons donc $f\left(x_{n}\right) \geqslant A$ pour $n=1,2,3, \ldots$ et $f^{\prime}(x)<A$, d'où il résulte tout de suite que

$$
\lim _{n=\infty} \frac{f\left(x_{n}\right)-f(x)}{x_{n}-x}=+\infty
$$

donc que $f_{+}^{\prime}(x)=+\infty$, c'est-à dire que $x$ appartient à $D$.

Les points $x$ n'appartenant pas à $E$ qui sont points d'accumulation de $E$ de côté droit (c'est-à dire qui sont limites de suites décroissantes de points de $E$ ) forment donc un ensemble au plus dénombrable $D_{1}$ (contenu dans $D$ ). Or les points qui sont points d'accumulation de $E$ de gauche, sans l'être de droite, forment, comme on sait, un cnsemble au plus dénombrable $D_{2}$. L'ensemble $E+D_{1}+D_{2}$ sera évidemment fermé. Il en résulte $\left(D_{1}+D_{2}\right.$ étant au plus dénombrable) que $E$ est un $G_{\dot{j}}$. Les ensembles $\mathrm{E}(f(x) \geqslant A)$ sont donc des $G_{\delta}$, d'où il résulte, comme on sait, que $f(x)$ est une fonction de classe $\leqslant 2$, c. q. f. d.

Théorème II: Si la dérivée $f_{+}^{\prime}(x)$ est presque partout finie, $f(x)$ est une fonction mesurable $(L)$.

La démonstration est tout à fait analogue à là précédente: on prouve que l'ensemble $\mathrm{E}(f(x) \geqslant A)+$ un ensemble de mesure nulle est fermé, d'où il résulte que $f(x)$ est mesurable $(L)$.

Comme conséquences immédiates nous obtenons les propositions:

Une fonction non mesurable $(B)$ a une dérivée de Dini infinie dans un ensemble non dénombrable de points.

Une fonction non mesurable $(L)$ a une dérivée de Divi infinie dans un ensemble de mesure positive ou dans un ensemble non mesurable (L). 